of a scanner. However, to assure that the data is read correctly they have to be assured by personnel viewing in addition to the programs own verifier program. The data is put in the database program Access and SPSS is used for statistical calculations. When the data of the patients is put into the database we get with the help of TELEFORM?s merging program new questionnaires for each patient to be used at the next registration.

Results We have until now about 280 patients registered for the first time and 55 of these are also registered for a second time. Conclusion

\section{OP0134 NONSTEROIDAL ANTIINFLAMMATORY DRUGS AND HOSPITALIZATIONS FOR GASTROINTESTINAL COMPLICATIONS IN CHINA}

${ }^{1} \mathrm{SL}$ Chen, ${ }^{2} \mathrm{SX}$ Kong, ${ }^{1} \mathrm{CD}$ Bao, ${ }^{2} \mathrm{P}$ Mavros, ${ }^{1} \mathrm{~S} C$ Chen, ${ }^{1} \mathrm{~J}$ Pei. ${ }^{1}$ Department of Rheumatology, Ren Ji Hospital, Shanghai, China; ${ }^{2}$ Outcomes Reseacrh, Merck \& Co., Inc., Whitehouse Station, NJ, USA

\subsection{6/annrheumdis-2001.859}

Background It has been well established that nonsteroidal antiinflammatory drugs (NSAID) cause gastrointestinal (GI) adverse events, ranging from minor symptoms to major complications leading to hospitalisation or death.

Objectives Assess the association between NSAID use and GI hospitalizations in China.

Methods The Shanghai Ren Ji Hospital is a tertiary medical centre located in central Shanghai, China. With 700 beds, the hospital has about 10,000 admissions per year. We identified cases from the hospital medical records from 1995 to 1999 based on modes of presentation [acute abdominal pain, vomiting, hematemesis, melena, shock (pulse $>100 \mathrm{bpm} /$ systolic $<100$ $\mathrm{mmHg}$ ), asymptomatic anaemia, other GI problems, or reason unknown with a discharge diagnosis related to GI complications]. One control was identified for each case, matched by age, sex, and admission date. Data were collected on demographics, history, diagnoses and treatment during the hospitalisation from the medical records. NSAID exposure was determined by drug use during the 1-year period prior to admission noted by the admitting physician. Logistic regression analysis was used to determine the association between NSAID use and GI complications after adjusting for age, sex, marital status, smoking history, and prior GPA use.

Results 939 patients with upper GI problems were identified as cases and of these 435 had upper GI complications as the primary diagnosis. 939 patients admitted without any GI problems were identified as controls. The mean age was 53 years and $87 \%$ were male in both groups. During the 1 -year period prior to admission, cases were more likely to be NSAID users than controls $(8.5 \%$ vs. $5.0 \%, \mathrm{p}<0.05)$. Similarly, use of gastroprotective agents (GPA, proton pump inhibitors and $\mathrm{H} 2$-antagonists) in the same time period was higher among cases than controls $(35.9 \%$ vs. $0.6 \%, p<0.05)$. Based on an analysis of the 939 cases and the corresponding controls, the odds ratio (OR) for GI hospitalizations of NSAID users versus nonusers adjusted by age, sex, marital status and history of smoking were 1.9 (95\% CI: 1.3-2.7). Similar analysis using only the 435 cases with upper GI complications as the primary diagnosis resulted in an OR estimate of 3.1 (95\% CI: 2.1-4.8). Adjusting for GPA use prior to hospital admission did not change the results.

Conclusion Among patients hospitalised in Shanghai, GI cases were significantly more likely to have used NSAIDs in the prior year than were controls. This suggests that use of NSAIDs is a significant, independent risk factor for GI hospitalisation in Shanghai, as has been demonstrated in numerous studies in developed countries.

\section{OP0135 HEALTHCARE RESOURCE USE AMONG USERS OF NONSTEROIDAL ANTI-INFLAMMATORY DRUGS IN ITALY}

${ }^{1} \mathrm{AP}$ Caputi, ${ }^{1} \mathrm{~A}$ Russo, ${ }^{2} \mathrm{SX}$ Kong, ${ }^{2} \mathrm{P}$ Mavros, ${ }^{3} \mathrm{E}$ Ricci. ${ }^{1}$ Institute of Pharmacology, University of Messina, Messina, Italy; ${ }^{2}$ Outcomes Research, Merck \& Co., Inc., Whitehouse Station, NJ, USA; ${ }^{3}$ Center for Health Economics, Institute Di Ricerche Farmacologiche, Ranica, Italy

\subsection{6/annrheumdis-2001.860}

Background Severe upper gastrointestinal (GI) complications such as perforation, ulcer or bleeding are associated with the use of nonsteroidal antiinflammatory drugs (NSAIDs). Most adverse drug reactions (ADRs) related to NSAIDs are non-life threatening, yet they represent a considerable amount of healthcare resource use.

Objectives Evaluate the use of health care resources associated with GI complications among NSAID users in Italy.

Methods From December 1998 to June 1999, 103 general practitioners in the Sicily region of Italy were asked to record data in 10 consecutive days on all patients who visited their offices. Physicians were instructed to fill out data collection forms regarding the type and indication of NSAIDs used and the use of medical resources (eg physician visits, specialist consultations, diagnostic tests, and hospitalizations) related to NSAIDs in general practice for the 6-month period prior to the visit. Physicians were also asked to indicate whether any patient visits during the past six months were related to NSAID ADRs.

Results A total of 913 NSAID users were identified among 20,668 patients who visited the physicians' offices. The mean age of the patients was 61 years and $61 \%$ were females. About $47 \%$ filled more than one NSAID prescription during the 6month period prior to the visit. Of all NSAID users, 50.3\% used at least one GI protective agent (including proton pump inhibitors, H2-antagonists, misoprostol and prescription or OTC antacids). There were on average five physician visits per patient. Of all physician visits, $49 \%$ were for the pathology for which an NSAID was prescribed, and 5\% were due to NSAID ADRs experienced by $23 \%$ of the patients. NSAID ADR occurrence was significantly associated with the number of NSAID prescriptions filled (OR: 1.5; 95\% CI: 1.3-1.8). The most common NSAID ADRs were upper abdominal pain in $13 \%$ of the patients, heartburn in $9 \%$, and dyspepsia in $2 \%$, while other NSAID ADRs occurred in $2 \%$ of the patients. Among all NSAID users, $4 \%$ had a GI endoscopic exam, 20\% had a specialist consultation, 5\% were hospitalised, and $31 \%$ had an X-ray. Compared to the patients who did not report any visits due to NSAID ADRs, those with NSAID ADRs were more likely to have a GI endoscopic exam (OR: 13.7; 95\% CI: 6.4-29.3), a specialist visit (OR: 2.0: 95\% CI: 1.4-2.9), hospitalisation (OR: 2.0; 95\% CI: 1.1-3.8), and X-ray (OR: 1.4; 95\% CI: 1.0-2.0).

Conclusion NSAID related adverse drug reactions were relatively common in this group of NSAID users and resulted in the consumption of a considerable amount of healthcare resources, such as physician visits, specialist consultations, diagnostic tests, and hospitalizations. 